\title{
The Use of a Compression Device as an Alternative to Hand-Sewn and Stapled Colorectal Anastomoses: Is Three a Crowd?
}

\author{
Brian S. Buchberg $\cdot$ Hossein Masoomi • \\ Herlinda Bergman • Steven D. Mills • Michael J. Stamos
}

Received: 10 September 2010 / Accepted: 19 October 2010/Published online: 10 November 2010

(C) 2010 The Author(s). This article is published with open access at Springerlink.com

\begin{abstract}
Background The NiTi CAR ${ }^{\mathrm{TM}} 27$ is a newer device that uses compression to create an anastomosis. An analysis of this device in the creation of colorectal anastomoses in humans has yet to be reported in the USA.

Methods A non-randomized, prospective pilot study of the NiTi CAR ${ }^{\mathrm{TM}} 27$ device in patients undergoing a left-sided colectomy between March 2008 and August 2009 was performed.

Results Twenty-three patients ( 9 men and 14 women) underwent a left-sided colectomy and compression anastomosis with the CAR ${ }^{\mathrm{TM}} 27$ device. Minor morbidities, 3 of 23 (13\%) patients, included one small postoperative abscess requiring antibiotics alone and two postoperative anastomotic strictures requiring balloon dilation. Major morbidities, 1 of 23 (4\%) patients, included a partial anastomotic dehiscence/leak requiring surgical dismantling of the anastomosis and diversion. Conclusion The CAR ${ }^{\mathrm{TM}} 27$ device shows promise as a safe and effective alternative for the creation of colorectal anastomoses. However, studies in a larger patient population are warranted to demonstrate equivalence of this device.
\end{abstract}

Keywords Anastomosis - Anastomotic leak .

Compression $\cdot$ Colorectal $\cdot$ Stricture $\cdot$ Nitinol

\section{Introduction}

Currently, the two available methods used for the creation of colorectal anastomoses include hand suturing and stapling devices. Although both are well established, they are not without their faults. Neither provides an immediately "sealed" anastomosis and both are prone to uncommon but serious complications such as anastomotic bleeding, strictures, or leaks. After colorectal resection, the incidence

Presented as part of an oral presentation session at the 62nd Annual Meeting of The Southwestern Surgical Congress held March 21-24, 2010, at the Loews Ventana Canyon Resort in Tucson, AZ.

B. S. Buchberg • H. Masoomi · H. Bergman · S. D. Mills •

M. J. Stamos $(\bowtie)$

Department of Surgery,

University of California-Irvine Medical Center,

333 City Blvd. West, Suite 700,

Orange, CA 92868, USA

e-mail: mstamos@uci.edu of anastomotic leak ranges from $2.9 \%$ to $15.3 \%,{ }^{1}$ while the incidence of subsequent stenosis or stricture ranges from $1.2 \%$ to $4.2 \%$. $^{2}$ These complications may require further surgical intervention and can lead to significant morbidity and mortality.

As a result, the concept of tissue compression during colorectal anastomoses has been revisited. This idea is not new and previous attempts have included the Murphy button, the biofragmentable anastomotic ring, and AKA-2 devices. ${ }^{3-11}$ Drawbacks of these devices included retained foreign material within the tissue, narrowing of the lumen, necrosis at the anastomotic site, and problems with passage of the deployment device. ${ }^{3}$ As a result, the use of these devices became extremely limited and was eventually replaced with the more reliable stapling devices.

Recently, the FDA-approved NiTi CAR ${ }^{\mathrm{TM}} 27$ (endoluminal compression anastomosis ring) device has been introduced and may overcome many limitations of the previous compression devices. The device is intended for use in the colon and rectum for the creation of end-to-end, end-to-side, and side-to-side anastomoses in both open and laparoscopic surgeries. This novel device consists of two disposable rings that trap the ends of transected bowel, 
bringing them into opposition and is intended for the creation of intestinal compression anastomoses in colorectal surgery in both open and laparoscopic surgeries. These compression anastomosis rings, composed of a nitinol alloy, exhibit super elasticity and shape memory to provide uniform compression to the tissue resulting in a secured, immediately sealed anastomosis. This translates, in animal models, to anastomoses with higher bursting strength and less stricture formation. ${ }^{12}$ Early research with the device in vitro, ex vivo, and in animal experiments suggests a safe and effective anastomosis when compared to that produced by staplers and sutures. Available clinical data suggest that the NiTi CAR ${ }^{\mathrm{TM}} 27$ device may address some of the limitations of the current methods for creating colorectal anastomosis. $^{13}$ Our plan was to evaluate the safety, technical feasibility, and effectiveness of the NiTi CAR ${ }^{\mathrm{TM}}$ 27 device in the creation of left-sided colorectal or colocolonic end-to-end compression anastomoses.

\section{Methods}

\section{Design and Study Population}

A non-randomized, prospective pilot study sponsored by NiTi $^{\mathrm{TM}}$ Surgical Solutions, Ltd. (Netanya, Israel) was undertaken to evaluate the outcomes of the NiTi CAR ${ }^{\mathrm{TM}}$ 27 compression anastomosis device in patients undergoing a left-sided colectomy between March 2008 and August 2009. Patients with either benign or malignant disease requiring a non-emergent open or laparoscopic colorectal resection with a high colorectal anastomosis $(\geq 10 \mathrm{~cm}$ from the anal verge) were eligible for trial inclusion. Patients with a known allergy to nickel were excluded from the study. Informed consent was obtained from all patients involved in the trial and approval for this trial was obtained from the Institutional Review Board at University of California-Irvine Medical Center.

\section{Clinical Parameters and Study Outcomes}

Clinical endpoints to be evaluated included intra- and postoperative complication rates, patient recovery, and anastomotic integrity. More specifically, the primary study outcome was the anastomotic leak rate. Anastomotic leakage was defined as clinical symptoms such as fever or sepsis in combination with abdominal/pelvic abscess, rectovaginal or colocutaneous fistula, or peritonitis within 30 days postoperatively, leading to a clinical and /or radiological interventional procedure of the subject or operation that confirms the leakage. ${ }^{14}$ Secondary study outcomes included time to return of bowel function as indicated by first postoperative passage of flatus or bowel movement, first postoperative toleration of liquids and solids, intraoperative device failure (i.e., cases of conversion to stapled or sutured anastomosis that are device related), presence of bleeding or stricture (either clinical evidence of a stricture or the inability to pass a sigmoidoscope through the anastomosis on postoperative follow-up), septic complications (i.e., wound infection, abscess formation, or peritonitis), and readmission, reoperation, or death within 30 days of the procedure. Length of time of the surgical procedure as well as ring expulsion time and awareness were also recorded for each patient.

\section{Device Description and Function}

The CAR-27'M device is similar to a regular circular stapler. It comprised two main parts, an applier and an implanted compression element. The compression element is composed of a plastic anvil ring and a metal ring, including shape memory nickel-titanium alloy (nitinol) leaf springs. When "fired," the device holds the two ends of tissue together with circumferentially placed barbed points, which penetrate through the tissue, holding it to the plastic ring. The nickel-titanium ring is released, creating equal compression both radially and longitudinally around the ring. The device has a circular blade which cuts the tissue within the ring, creating a patent anastomosis. The tissue heals around the circular edges that are held within the ring and the device along with the compressed tissue slough off over the following $8-10$ days, at which point the ring is expelled from the body with a later bowel movement (Figs. 1 and 2). The result is a full circumferential, hemostatic sealed anastomosis without any retained foreign material (Fig. 3).

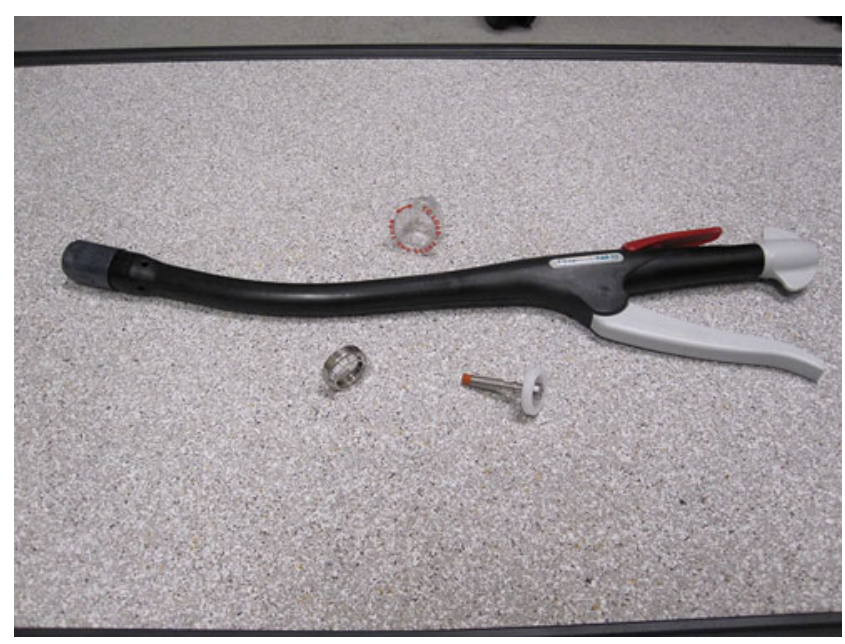

Fig. 1 NiTi CAR ${ }^{\mathrm{TM}} 27$ compression anastomosis device. Components include the firing instrument, ring loader, anvil, nitinol metal compression ring 


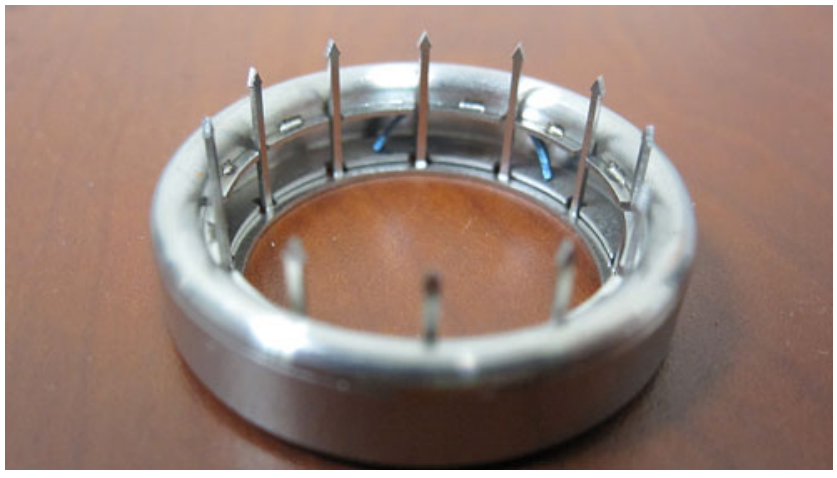

Fig. 2 Compression anastomosis ring $(C A R)$ containing shape memory nickel-titanium alloy springs inside a ring with barbs allowing for equal compression around the ring. The device, along with the compressed tissue, sloughs off over the following 8-10 days, at which point the ring is expelled from the body with a later bowel movement

\section{Study Procedures}

\section{Pre-surgery}

Procedures performed such as routine hospital examinations, mechanical bowel preparation, and antibiotic prophylactic treatment were completed according to our standard management protocol. During preoperative surgical evaluation for each patient, the following information was recorded: demographic data, ASA status, diagnosis leading to surgery, relevant surgical and medical comorbidities, and current treatments and medications received in the last 6 months. Preoperative protocol for all enrolled patients included completion of a routine bowel preparation the day prior to surgery in combination with a clear liquid diet. Stool softeners were provided for all patients before and after the surgical procedure to avoid hard stools, which may exert an undesirable mechanical force on the anastomotic area while the ring is still in place. Additionally, a single dose of prophylactic, broad-spectrum intravenous antibiotics was administered $<60 \mathrm{~min}$ prior to incision.

Intraoperative

\section{Surgeon Use of Device}

All procedures were performed by one of two boardcertified colorectal surgeons at our institution. To ensure that the surgeons were competent with using the device prior to participation in the study, each was trained in the use of the device on a porcine model. The device functions almost identically to the current circular staplers in widespread use, facilitating the minimal "learning curve." All surgeons went through a review of the procedure using the CAR ${ }^{\text {TM }} 27$ prior to the study. The device was used according to the manufacturer's guidelines. Initially, the surgeon divided the colon proximal to the diseased colon to place a purse string and also divided the colon or rectum distal to the diseased intestine using a linear stapler (from within the abdomen). To create the anastomosis, several steps were performed:

Prior to loading the CAR, the alloy ring was cooled by immersing in a bowl of sterile, cold water for at least $5 \mathrm{~min}$. The detached anvil head of the device was inserted into the proximal colon and secured with a purse-string suture. One of the surgeons then inserted the device body carefully through the anal canal. The compression ring housing (with sharp point) was advanced (preferably near the center of the staple line) and the trocar (spike) was exposed via a counter clockwise rotation of the device (almost identical to a standard circular stapling device). The two parts of the device and bowel were then attached - the detachable anvil head secured with purse string and the trocar side. After a

Fig. 3 Fully circumferential, hemostatic sealed anastomosis without any retained foreign material seen on flexible sigmoidoscopy at 3-month follow-up

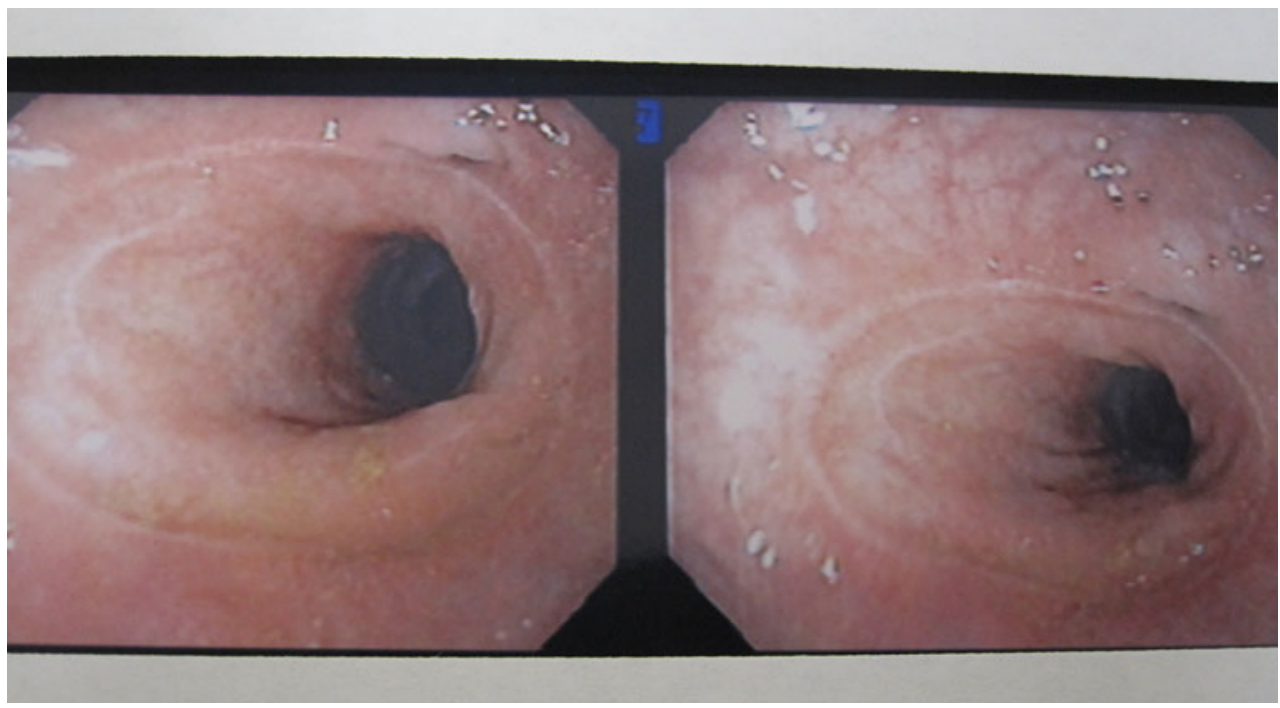


clockwise closure of the two device parts, the firing mechanism was triggered, creating the anastomosis. The anastomosis height was measured and recorded in the patient's case report form (CRF). The device rings created the anastomosis, and a circular rim or donut of tissue from the proximal and distal margins was removed with the device. Approximating body temperature, the ring recovers its programmed shape, applying pressure on the tissue. After removal of the device, the integrity of the anastomosis was checked by direct palpation from the abdominal side. Insufflation of air via proctoscope was performed with the pelvis filled with saline to verify the absence of leak. The anastomotic donuts were also checked for integrity.

Intraoperative variables recorded included: date and duration of surgery, ASA status, estimated blood loss, and CAR lot number; type of operation (open, laparoscopic), laparoscopic conversion, and if drains were used (number and type); and ease of CAR deployment ( $1=$ very difficult to $5=$ very easy) and technical complications. Additional recorded information included the presence or absence of leak detected with air testing, concomitant procedures performed during surgery, use of a diverting ileostomy, and other procedural complications.

\section{Postoperative Follow-Up}

Follow-up evaluations were performed every day while patients were hospitalized. The following information was recorded for all patients while they were hospitalized (until discharge or postoperative day 20): passing flatus and bowel movements, tolerating liquids and solids, and temperature (max. for 24-h period), ring expulsion if it occurred during this time, and date of discharge.

There were two postoperative clinic visits: $\leq 1$ month and a 3-month ( \pm 2 weeks) visit which included a proctoscopic exam of the anastomotic site during one of the visits (performed at the first visit per standard of care if symptoms suggested stricture and at the second visit for the same reasons or as routine protocol even in the absence of symptoms). Information concerning bowel movements, liquid and solid tolerability, temperature, and when ring passage occurred was collected.

\section{Results}

A total of 23 patients ( 9 men and 14 women) underwent a left-sided colectomy and subsequent compression anastomosis (12 laparoscopic and 11 open) with the CAR ${ }^{\text {TM }} 27$ device. Age at operation ranged from 34 to 78 (mean age, 60.0 years) and BMI ranged from 20.0 to 36.5 (mean BMI, 26.0). A majority of the patients were Caucasian (78\%). The ASA classification for the population was: ASA II,
$65 \%$ and ASA III, 35\%. The most common patient comorbidities included hypertension (57\% of patients), malignancy $(26 \%)$, obesity $(22 \%)$, diabetes mellitus (17\%), and coronary artery disease (13\%). Diagnoses leading to surgery included rectal prolapse (eight patients), chronic diverticulitis (sux patients), diverticular colonic fistula (five patients), and malignancy (four patients; Table 1). Of note is that one patient had a sigmoid colectomy for diverticulitis with a concomitant distal pancreatectomy performed by a pancreatic surgeon for a previously diagnosed neuroendocrine tumor.

The duration of surgery range for this patient population was 75-330 min (median, $149 \mathrm{~min}$ ). Estimated blood loss ranged from 20 to $700 \mathrm{~mL}$ (median, $150 \mathrm{~mL}$ ), and none of the 12 laparoscopic procedures required conversion to an open operation. For each of the 23 anastomoses, the average time for creation was $5.7 \mathrm{~min}$ (range, 3-17 $\mathrm{min}$ ). The ease of CAR deployment, assessed by the operating surgeon, was also recorded $(1=$ very difficult to $5=$ very easy). Overall, the operating surgeons thought that the CAR ${ }^{\mathrm{TM}} 27$ device was easy to use (mean score, 4.5 ). The score was 1 (very difficult) in $4.3 \%$ of patients, 3 (difficult) in $4.3 \%$, 4 (easy) in $26.1 \%$, and 5 (very easy) in $65.2 \%$ of patients. The reason for a lower score ( 3 or less) in two patients was failure of the device to fire appropriately on first attempt. No case had to be converted to a stapled or hand-sutured anastomosis. The distance from the anal verge to the anastomosis ranged from 9 to $20 \mathrm{~cm}$ (mean distance, $14 \mathrm{~cm}$ ).

The median time to return of bowel function as indicated by the passage of flatus was 4 (range, 2-31)days. The median length of hospital stay was 5 (range, 3-41)days, and the median postoperative follow-up was 91 (range, 14-214)days. Of note is that one patient failed to return for 1- and 3-month follow-up visits. Time to ring expulsion was difficult to measure since most patients were surprisingly unaware of this event. However, one patient stated that the ring passed without complication on the tenth postoperative day.

Overall, surgical complications were grouped into two categories: (1) minor morbidities, 3 of 23 (13\%) patients, which included one small postoperative abscess requiring antibiotics alone and two postoperative anastomotic strictures (diagnosed by flexible sigmoidoscopy at 3-month visit) receiving balloon dilation and (2) major morbidities, 1 of $23(4 \%)$ patients, which included a partial anastomotic dehiscence/leak requiring a return to the operating room for dismantling of the anastomosis and diversion. The patient had a prolonged hospital course but slowly improved and was discharged home on postoperative day (POD) 41 tolerating a regular diet. Of note is that air testing for anastomotic integrity just after completion of the anastomosis did not demonstrate a leak in any patient.

At 1-month follow-up, all patients were tolerating a regular diet and all except one patient reported having 
Table 1 NiTi patient characteristics

$C O P D$ chronic obstructive pulmonary disease, $H T N$ hypertension, $D M$ diabetes mellitus, $C A D$ coronary artery disease, $A S A$ American Society of Anesthesiologists

\begin{tabular}{|c|c|c|c|}
\hline Race & ASA score & BMI & Major comorbidities \\
\hline Caucasian & 3 & 26 & COPD \\
\hline Caucasian & 2 & 24.8 & Hypothyroidism \\
\hline Caucasian & 3 & 36.5 & HTN, DM, obesity \\
\hline Hispanic & 2 & 24 & HTN \\
\hline Caucasian & 3 & 23.9 & CAD, HTN \\
\hline Caucasian & 2 & 23 & HTN, testicular cancer \\
\hline Caucasian & 3 & 34.5 & $\mathrm{DM}$, CAD, obesity, breast cancer \\
\hline Asian & 2 & 23.1 & HTN, gout \\
\hline Caucasian & 2 & 30.2 & HTN, obesity \\
\hline Caucasian & 2 & 20.3 & Tobacco use \\
\hline Caucasian & 2 & 26.6 & HTN \\
\hline Caucasian & 3 & 22.8 & HTN, DM, asthma \\
\hline Caucasian & 2 & 25.8 & HTN \\
\hline Caucasian & 2 & 20.7 & - \\
\hline Caucasian & 2 & 20.4 & Tobacco use \\
\hline Hispanic & 2 & 34 & Obesity \\
\hline Asian & 2 & 26.2 & HTN, DM \\
\hline Caucasian & 3 & 22.8 & HTN, CAD \\
\hline African-American & 2 & 26.7 & HTN, asthma \\
\hline Caucasian & 3 & 33.2 & Obesity \\
\hline Caucasian & 2 & 26.3 & HTN, atrial fibrillation \\
\hline Caucasian & 2 & 20 & Hypothyroidism \\
\hline Caucasian & 3 & 21 & Carotid artery stenosis \\
\hline
\end{tabular}

normal bowel movements. This patient had intermittent episodes of loose bowel movements prior to operation, most likely as a result of rectal prolapse. Postoperatively, the patient continued to have intermittent episodes of loose bowel movements with improved frequency that finally resolved by the 3-month follow-up visit. At 3-month follow-up, all patients were tolerating a regular diet and all reported having normal bowel movements, except two patients who reported mild abdominal pain and bowel movements with a string-like consistency. Flexible sigmoidoscopy of the anastomotic sites of both patients performed at this time revealed tight strictures. Both patients subsequently underwent endoscopic balloon dilation to resolve their strictures and both have made full recoveries. Flexible sigmoidoscopy of all remaining patients was unremarkable (Table 2).

\section{Discussion}

Use of the newer CAR ${ }^{\mathrm{TM}} 27$ compression anastomotic device has the advantage of overcoming several obstacles previously encountered with earlier compression devices. Older devices resulted in the creation of lumen too narrow for the passage of intestinal contents, retention of foreign material within the tissue, necrosis at the anastomotic site, and problems with passage of the device. The CAR ${ }^{\mathrm{TM}} 27$ device has an anatomical design not unlike that of current stapling devices used to create end-to-end anastomoses and therefore eliminates the previous problems of passage and withdrawal of the device from the anus. Compression of the two ends of bowel to be connected creates a controlled region of necrosis isolated within the compression ring only, allowing a more rapid healing outside the ring. Because the ring is expelled early in the healing process, retention of foreign material ceases to be an issue. In addition, the CAR ${ }^{\mathrm{TM}} 27$ device has potential advantages over current methods of large bowel anastomosis, most notably the lag phase of anastomotic healing. ${ }^{13}$ The tissue compression exerted through the CAR ${ }^{\mathrm{TM}} 27$ device provides a strong seal circumferentially immediately at the time of surgery secondary to the exhibition of super elasticity and shape memory, and the ability to eliminate the permanent implantation characteristics inherent in stapled anastomosis by the nitinol-based compression ring. In addition, the longitudinally orientated metal prongs further fixate both bowel ends and prevent tissue slippage from axial movements. The overall goal of such a design is the creation of a safe and effective anastomosis with mitigation of complications commonly seen with current methods of large bowel anastomosis, namely leaks, strictures, and bleeding. 
Table 2 NiTi patient outcomes

\begin{tabular}{|c|c|c|c|c|c|c|}
\hline Diagnosis & Lap/open & $\begin{array}{l}\text { Return of bowel } \\
\text { function (days) }\end{array}$ & $\begin{array}{l}\text { Immediate } \\
\text { postoperative } \\
\text { complications }\end{array}$ & $\begin{array}{l}\text { Length of } \\
\text { stay (days) }\end{array}$ & $\begin{array}{l}\text { Follow-up } \\
\text { complications }\end{array}$ & $\begin{array}{l}\text { Flexible } \\
\text { sigmoidoscopy } \\
\text { (3 months' visit) }\end{array}$ \\
\hline Rectal prolapse & Open & 3 & None & 5 & None & Normal \\
\hline Rectal prolapse & Open & 7 & None & 8 & None & Normal \\
\hline Rectal prolapse & Open & 4 & UTI & 11 & None & Normal \\
\hline Coloenteric fistula & Lap & 4 & Abscess & 12 & None & Normal \\
\hline Diverticulitis & Lap & 3 & $\begin{array}{l}\text { Improper firing } \\
\text { on first attempt }\end{array}$ & 4 & None & Normal \\
\hline Sigmoid cancer & Lap & 2 & None & 4 & None & Normal \\
\hline Colovaginal fistula & Open & 6 & Wound infection & 6 & Wound infection improved & Normal \\
\hline Sigmoid cancer & Lap & 7 & None & 8 & None & Normal \\
\hline Diverticulitis & Open & 5 & Pancreatic leak ${ }^{\mathrm{a}}$ & 9 & None & No Follow up \\
\hline Diverticulitis & Lap & 2 & None & 7 & None & $\begin{array}{l}\text { Stricture } \\
\text { (Balloon dilation }\end{array}$ \\
\hline Colovaginal fistula & Open & 5 & None & 6 & None & $\begin{array}{l}\text { Stricture } \\
\text { (Balloon dilation) }\end{array}$ \\
\hline Rectal cancer & Lap & 4 & None & 7 & None & Normal \\
\hline Rectal prolapse & Open & 2 & None & 4 & Diarrhea & Normal \\
\hline Rectal prolapse & Open & 3 & $\begin{array}{l}\text { Improper firing } \\
\text { on first attempt }\end{array}$ & 3 & None & Normal \\
\hline Rectal prolapse & Open & 4 & None & 4 & None & Normal \\
\hline Diverticulitis & Lap & 3 & None & 6 & None & Normal \\
\hline Sigmoid cancer & Lap & 3 & None & 4 & Readmit-dehydration & Normal \\
\hline Colovesicular fistula & Lap & 2 & None & 4 & Wound abscess & Normal \\
\hline Colonic fistula & Lap & 2 & None & 4 & None & \\
\hline Rectal prolapse & Open & 4 & None & 6 & Intermittent-incontinence & Normal \\
\hline Diverticulitis & Lap & 2 & None & 3 & None & Normal \\
\hline Rectal prolapse & Open & 2 & None & 3 & & Normal \\
\hline Diverticulitis & Lap & 31 & Anastomotic disruption & 41 & $\begin{array}{l}\text { Hartmann's pouch } \\
\text { and colostomy }\end{array}$ & $\mathrm{N} \backslash \mathrm{A}$ \\
\hline
\end{tabular}

Lap laparoscopic

${ }^{a}$ This patient had a distal pancreatectomy for a neuroendocrine tumor at the same time as colon resection

Improvements in these outcomes have been observed in earlier studies with the CAR ${ }^{\mathrm{TM}} 27$ device on human subjects. D'Hoore et al. ${ }^{13}$ demonstrated early promising findings with the CAR ${ }^{\mathrm{TM}} 27$ device. In ten patients who underwent leftsided colectomy, no anastomotic leakage occurred, median hospital stay was 4 days, and only three patients noticed passage of the ring. Ring passage was verified with a plain abdominal X-ray 3 weeks after operation. Similar findings were reported in 12 patients after use of the compression device resulting in no complications related to the anastomosis, with only two patients noticing ring passage. ${ }^{15}$

In comparison, our study involving 23 patients resulted in an anastomotic leak in one patient $(4.3 \%)$ and stricture formation in two patients $(8.6 \%)$. The patient with the anastomotic leak required complete dismantling of the anastomosis and creation of an end colostomy. Investigation into possible reasons for anastomotic failure initiated by the operating surgeon and the device manufacturer failed to elucidate any definitive cause. The early presentation of the leak (POD 4) and the intraoperative findings suggested that premature necrosis of the tissue may be a contributor. This patient's preexisting comorbidities (i.e., diverticulitis and moderate malnutrition) may also have been a contributing factor. In the two patients who developed strictures, one possible explanation was a retained ring for a longer duration than is typically experienced, inciting an ongoing inflammatory response causing scarring. Although, since neither patient noticed ring passage, this is only conjecture. Fortunately, balloon dilation easily resolved their symptoms.

Overall the operating surgeons felt that the device was easy to use; however, in two different patients, device failures occurred. In both instances, the circular blade within the device that cuts the tissue within the ring malfunctioned, preventing removal of the anvil from the anastomotic site. 
This required manual withdrawal of the anvil and device, resection of this anastomotic site, and creation of a new anastomosis. The second attempt was successful for each patient and neither experienced further sequelae. Time to creation of anastomosis was relatively short, as was time to return of bowel function and length of hospital stay, further enhancing the attractiveness of this device. Only one patient noticed passage of the ring, indicating no postoperative concern by both patient and surgeon for retained foreign objects. Furthermore, flexible sigmoidoscopy performed at 3 months postoperatively confirmed a well-healed anastomosis and verified ring passage.

The present study consists of a small number of patients using a new compression anastomotic device to create colorectal anastomoses. Although there was only one definitive anastomotic leak translating to a $4.3 \%$ leak rate for high rectal anastomoses, such a small sample size makes it impossible to draw firm conclusions regarding the efficacy and safety of the CAR ${ }^{\mathrm{TM}} 27$ device. This leak rate is within the range expected for this level of anastomosis (3-5\%). ${ }^{16-18}$ Additionally, the two strictures encountered, although easy to manage, raise questions regarding the frequency of this complication relative to current stapled anastmoses. Finally, although the two "misfirings" of the device are troubling, they may be related to the learning curve and therefore can be avoided with further training. Larger, randomized trials with a head-to-head comparison to stapled and/or hand-sewn anastomoses will be necessary to accurately assess this new technique.

Open Access This article is distributed under the terms of the Creative Commons Attribution Noncommercial License which permits any noncommercial use, distribution, and reproduction in any medium, provided the original author(s) and source are credited.

\section{References}

1. Chang, SC, Lin, JK, Yang, SH, Jiang, JK, Chen, WC, Lin, TC. "Long term outcome of anastomotic leakage after curative resection for mid and low rectal tumors" 2003. Hepatogastroenterology 50: 1898-902.
2. E. Rullier, C. Laurent and J.L. Garrelon et al., Risk factors for anastomotic leakage after resection of rectal cancer, 2004. $\mathrm{Br} \mathrm{J}$ Surg 85, 355-358.

3. Aggarwal R, Darzi A. Compression anastomoses revisited. J Am Coll Surg 2005; 201(6):965-71.

4. Chua CL. Colonic anastomosis with sutureless biofragmentable rings. Singapore Med J 1993; 34(6):541-4.

5. Di Castro A, Biancari F, Brocato R, et al. Intestinal anastomosis with the biofragmentable anastomosis ring. Am J Surg 1998; 176 (5):472-4, 7 .

6. Konishi F, Saito Y, Ugajin H, et al. Sutureless anastomosis using a biofragmentable anastomosis ring. Surg Today 1995; 25(9):783-9.

7. Melzer A, Stockel D. Using shape-memory alloys. Med Device Technol 1995; 6(4):16-20, 22-3.

8. Rutkow IM. John Benjamin Murphy, 1857-1916. Arch Surg 2001; 136(3):359.

9. Thiede A, Geiger D, Dietz UA, et al. Overview on compression anastomoses: biofragmentable anastomosis ring multicenter prospective trial of 1666 anastomoses. World J Surg 1998; 22(1):7886; discussion 87

10. Valle M, Biancari F, Caviglia A, et al. The biofragmentable anastomosis ring in elective colon resections. Int Surg 1998; 83 (1):58-9.

11. Wullstein C, Gross E. Compression anastomosis (AKA-2) in colorectal surgery: results in 442 consecutive patients. Br J Surg 2000; 87(8):1071-5.

12. David Stewart, Steven Hunt, Richard Pierce, Dongli Mao, Margaret Frisella, Kathryn Cook, Barry Starcher and James Fleshman Validation of the NITI Endoluminal Compression Anastomosis Ring (EndoCAR) Device and Comparison to the Traditional Circular Stapled Colorectal Anastomosis in a Porcine Model Surg Innov 2007; 14; 252.

13. D'Hoore A, Hompes D, Folkesson J, et al. Circular 'superelastic' compression anastomosis: from the animal lab to clinical practice. Minim Invasive Ther Allied Technol 2008; 17(3):172-5.

14. Jestin P, Pahlman L, Gunnarsson U. Risk factors for anastomotic leakage after rectal cancer surgery: a case-control study. Colorectal Dis 2008; 10(7):715-21.

15. B. Dauser, F. Herbst. NITI Endoluminal Compression Anastomosis Ring (NITI CAR 27 ${ }^{\circledR}$ ): A breakthrough in compression anastomoses?. Eur Surg (2009) 41/3: 116-119.

16. Neil Hyman, MD, Thomas L. Manchester, MD, Turner Osler, MD, Betsy Burns, NP, and Peter A. Cataldo, MD. Anastomotic Leaks After Intestinal Anastomosis. It's Later Than You Think. Ann Surg. 2007 February; 245(2): 254-258

17. Detry RJ, Kartheuser A, Delriviere L, Saba J, Kestens PJ. Use of the circular stapler in 1000 consecutive colorectal anastomoses: experience of one surgical team. Surgery. $1995 \mathrm{Feb}$;117(2):140-5.

18. Wai Lun Law \& Hok Kwok Choi \& Yee Man Lee \& Judy W. C. Ho \& Chi Leung Seto. Anastomotic Leakage is Associated with Poor Long-Term Outcome in Patients After Curative Colorectal Resection for Malignancy. J Gastrointest Surg (2007) 11:8-15. 